\title{
Detection of solid ammonia, methanol, and methane with ISOPHOT`
}

\author{
J. Gürtler ${ }^{1}$, U. Klaas ${ }^{2}$, Th. Henning ${ }^{1,2}$, P. Ábrahám ${ }^{2,3}$, D. Lemke ${ }^{2}$, K. Schreyer ${ }^{1}$, and K. Lehmann ${ }^{4}$ \\ 1 Astrophysikalisches Institut und Universitäts-Sternwarte, Schillergäßchen 2-3, 07745 Jena, Germany \\ 2 Max-Planck-Institut für Astronomie, Königstuhl 17, 69117 Heidelberg, Germany \\ 3 Konkoly Observatory of the Hungarian Academy of Sciences, PO Box 67, 1525 Budapest, Hungary \\ ${ }^{4}$ Brunnersweg 12, 64380 Roßdorf, Germany
}

Received 30 March 2001 / Accepted 16 May 2002

\begin{abstract}
We report on a search for the $\mathrm{NH}_{3}$ inversion band at $9.0 \mu \mathrm{m}$, the $\mathrm{CH}_{4}$ band at $7.7 \mu \mathrm{m}$, and the $\mathrm{CH}_{3} \mathrm{OH}$ band at $9.7 \mu \mathrm{m}$ in the spectra of 10 heavily obscured stellar objects taken with the spectrophotometer ISOPHOT-S aboard the Infrared Space Observatory (ISO). Four of them show indications of the $\mathrm{NH}_{3}$ inversion band at $9.0 \mu \mathrm{m}$ superimposed on the always strong silicate band. The results show that ammonia ice may be abundant in some cool and dense molecular circumstellar regions. The $9.7 \mu \mathrm{m}$ band of solid $\mathrm{CH}_{3} \mathrm{OH}$ was detected in 5 of the sources, and also the $7.7 \mu \mathrm{m}$ band of solid $\mathrm{CH}_{4}$ was found in 5 sources. The PHT-S detections were cross-checked against ISO-SWS spectra from archive data. Considering the different beam sizes $\left(24 \times 24 \square^{\prime \prime}\right.$ versus $\left.14 \times 20 \square^{\prime \prime}\right)$, there is good agreement.
\end{abstract}

Key words. stars: circumstellar matter - ISM: dust, extinction - ISM: molecules - ISM: lines and bands - infrared: ISM

\section{Introduction}

The near- and mid-infrared spectral regions are crucial for the study of the composition of interstellar dust. Over the past 20 years, infrared spectroscopy from the ground, aircraft, and space has shown that interstellar grains in molecular clouds contain a variety of frozen molecules ("ices") in addition to refractory materials such as silicate and carbonaceous grains. A new era for dust spectroscopy was opened with the launch of the Infrared Space Observatory (ISO) (Kessler et al. 1996) with its unprecedented spectroscopic possibilities. For a summary of results see, e.g., d'Hendecourt et al. (1999) and Salama et al. (2000).

The molecular ices play a significant role in the chemistry of interstellar clouds. The icy grain mantles are both sinks and sources of molecules in the gas phase and formation place for particular molecular species. Therefore, it is desirable to have the inventory of molecular ices as complete as possible and to derive the abundances of the various ice components in different objects.

The most abundant ice component known is frozen $\mathrm{H}_{2} \mathrm{O}$, but solid $\mathrm{CO}$ and $\mathrm{CO}_{2}$ are ubiquitous, too (Whittet 1993; Gürtler et al. 1996; Whittet et al. 1996; Gerakines et al. 1999). Minor components are $\mathrm{CH}_{4}$ (d'Hendecourt et al. 1996;

Send offprint requests to: J. Gürtler, e-mail: guertler@astro.uni-jena.de

* Based on observations with ISO, an ESA project with instruments funded by ESA Member States (especially the PI countries: France, Germany, The Netherlands and the United Kingdom) with the participation of ISAS and NASA.
Boogert et al. 1996, 1998), $\mathrm{CH}_{3} \mathrm{OH}$ (Grim et al. 1991; Skinner et al. 1992; Dartois et al. 1999), a molecule or ion containing the CN group ("XCN"; Lacy et al. 1984; Grim \& Greenberg 1987; Demyk et al. 1998; Pendleton et al. 1999), and possibly HCOOH (Schutte et al. 1996).

As for the presence of solid ammonia, the situation is less clear. Submillimetre observations have shown gaseous $\mathrm{NH}_{3}$ to be an abundant molecule in interstellar clouds. Because it freezes out at the low temperatures prevailing in dense clouds (condensation/sublimation point $195 \mathrm{~K}$ under normal conditions), we may expect it to be a component of interstellar ices. Chemical models also suggest that it is readily formed on grain surfaces in environments rich in atomic hydrogen (e.g., Herbst 1993).

Solid $\mathrm{NH}_{3}$ has infrared active vibrational fundamentals at $2.95 \mu \mathrm{m}$ (N-H stretching), $6.16 \mu \mathrm{m}$ (N-H deformation), and $9.0 \mu \mathrm{m}$ (inversion). All of them overlap with other interstellar absorption bands.

The stretching band, which has been studied most, overlaps with the $\mathrm{O}-\mathrm{H}$ stretching band of $\mathrm{H}_{2} \mathrm{O}$ at $3.08 \mu \mathrm{m}$. Earlier detections in a number of sources (Hagen et al. 1980, Knacke et al. 1982) could not be confirmed by later observations (Knacke \& McCorkle 1987; Smith et al. 1989). A detection in HH 100-IRS by Graham \& Chen (1991) could not be confirmed by Whittet et al. (1996). In spectra taken with the Short Wavelength Spectrometer (SWS) of ISO Chiar et al. (2000) found clear indications of the $\mathrm{NH}_{3}$ stretching band in the profile of the water ice band of $\mathrm{Sgr} \mathrm{A}^{*}$ and two other Galactic Centre sources and derived an $\mathrm{NH}_{3}$ abundance of 20-30\% 
relative to water along these lines of sight. Dartois \& d'Hendecourt (2001), however, modelled the profile of the $3.08 \mu \mathrm{m}$ band in the direction of the Galactic Centre and several other directions without any admixture of $\mathrm{NH}_{3}$ and derived upper limits for the abundance of $\mathrm{NH}_{3}$ in $\mathrm{Sgr} \mathrm{A} *$ of $17 \%$.

The deformation band is the weakest of the three. It is of little astrophysical use because it overlaps with the $\mathrm{H}_{2} \mathrm{O}$ bending mode at $6.0 \mu \mathrm{m}$.

The inversion band falls within the broad and strong silicate feature. Lacy et al. (1998) reported the detection of a weak feature at $9.0 \mu \mathrm{m}$ in the spectrum of the deeply embedded infrared source NGC 7538:IRS9. Its shape could be fitted well with laboratory data for $\mathrm{H}_{2} \mathrm{O}$ ice containing 2-10\% ammonia, which argues in favour of the reality of the feature and its identification. Lacy et al. (1998) were unable to unambiguously detect the ammonia ice band in W33A and W3:IRS5. Based on observations with the Short Wavelength Spectrometer (SWS) of ISO, Gibb et al. (2000) presented a complete 2.4-25 $\mu \mathrm{m}$ spectrum of W33A. It clearly shows a feature around $9.0 \mu \mathrm{m}$, but they could not fit it well by a laboratory spectrum for $\mathrm{NH}_{3}$ ice. Recently Gibb et al. (2001) conducted a search for the $\mathrm{NH}_{3}$ inversion band in SWS spectra of young objects. They confirmed the earlier detections of the $\mathrm{NH}_{3}$ inversion band in NGC 7538:IRS9 and W33A and detected it in AFGL 989 and AFGL 7009S.

In this paper we report on a search for the $\mathrm{NH}_{3}$ inversion band in spectra of heavily obscured young stellar objects taken with the spectrophotometer ISOPHOT (Lemke et al. 1996) aboard the Infrared Space Observatory (ISO). We confirm the finding of Gibb et al. (2000), could not find a feature in NGC 7538:IRS9, and claim the detection of the band in the spectra of 3 additional sources. The detection of $\mathrm{NH}_{3}$ is of particular importance because it is a basic building block for more complex nitrogen compounds (Sandford et al. 1998). We also searched for the $9.7 \mu \mathrm{m}$ band of solid $\mathrm{CH}_{3} \mathrm{OH}$ and detected it in five sources, in one of them for the first time. Moreover, we searched for the $\mathrm{CH}_{4}$ deformation band at $7.7 \mu \mathrm{m}$. We detected it in five sources. To verify our detection method, we compare our results with SWS spectra if available.

The sources to be investigated were selected because their spectra show both a deep silicate band (measure for the amount of refractory dust) and the water ice band at $3.08 \mu \mathrm{m}$ (indicator of volatile grain mantles). Results for the $4.27 \mu \mathrm{m}$ band of solid $\mathrm{CO}_{2}$ were reported earlier by Gürtler et al. (1996).

\section{Observations and data reduction}

The observations were carried out using the spectrophotometer subsystem ISOPHOT-S. It consists of two low-resolution grating spectrometers covering the wavelength ranges $2.5-4.9 \mu \mathrm{m}$ (PHT-SS) and 5.8-11.6 $\mu \mathrm{m}$ (PHT-SL) and having a common entrance aperture of $24^{\prime \prime} \times 24^{\prime \prime}$. Each channel has a linear 64-element array of Si:Ga detectors. The spectral band width $(F W H M)$ of a single detector is $0.0383 \mu \mathrm{m}$ for PHT-SS and $0.0918 \mu \mathrm{m}$ for PHT-SL, resulting in a mean $\lambda / \Delta \lambda$ of about 95 for both channels. A more detailed description of ISOPHOT-S has been given by Klaas et al. (1997).

The observations were performed between February 4, 1996 and August 2, 1997. One source, HH 100-IRS, was observed twice with an interlude of about 1.5 years. The orientation of the aperture on the sky was quite similar.

The raw data were reduced using the standard procedures of the PHT Interactive Analysis (PIA) software (version 9.0) ${ }^{1}$. We used the so-called dynamic spectral response function calibration which takes care of signal transients during the exposure. The absolute photometric accuracy of this method is better than $10 \%$, the uncertainty of the relative spectral shape is better than $10 \%$, too (Klaas et al. 2000).

The SWS spectra presented here were retrieved from the ISO Data Archive (Kessler et al. 2000) after on-the-fly reprocessing with the automatic data reduction pipeline OLP V10.0 using the calibration data set CALG V7.0. The subsequent processing of the Auto Analysis Results of the SWS full spectral scans was done using ISAP ${ }^{2}$ (Sturm et al. 1998). In ISAP we followed the recipes given in Sturm (2000): bad data flagged by the OLP were removed during read-in. The data were split into the sub-spectra and only the relevant line tags 6 and 7 $(5.24-12.60 \mu \mathrm{m})$ were processed. After interactive removal of outliers or occasional signal sets belonging to noisy detectors, flat-fielding with the "preserving the mean" method was done. Afterwards the spectra of all 12 detectors were averaged and finally the resulting spectrum was smoothed with a Gaussian filter with a width of $0.096 \mu \mathrm{m}$ to adjust the resolution to the PHT-S spectra.

In Fig. 1 both sets of spectra are compared. The general agreement is good. Remarkable differences appear for W3:IRS5, W33 A, and possibly Cep A. Here, the SWS spectra show a deeper silicate band while the level of the continuum outside the silicate band appears only marginally lower. The main reason is the different size of the field of view of both instruments (280 $\square^{\prime \prime}$ versus $\left.576 \square^{\prime \prime}\right)$. In the case of AFGL 2591 the disagreement can be attributed to using deviating source positions during the observations. A more detailed discussion will be done in Sect. 4.

\section{Extraction of the molecular ice bands}

The main task in any search for the $\mathrm{NH}_{3}$ inversion band is the determination of the undisturbed silicate profile. Since the silicate profile is not the same for all sources, we cannot use a standard profile to extract any spectral band that may be superimposed. Neither is there any obvious way to model the actual silicate profile with the necessary accuracy by means of laboratory data.

We searched both the PHT-S and the SWS spectra by an analogous procedure. To minimize the necessary assumptions, we have resorted to a purely formal solution and approximated the profile of the silicate band by fitting polynomials to it. Indeed, this is the same procedure which was adopted by Lacy et al. (1998) and Gibb et al. (2000). The laboratory data of

\footnotetext{
1 PIA is a joint development by the ESA Astrophysics Division and the ISOPHOT consortium led by the Max-Planck-Institut für Astronomie (MPIA), Heidelberg.

2 The ISO Spectral Analysis Package (ISAP) is a joint development by the LWS and SWS Instrument Teams and Data Centers. Contributing institutes are CESR, IAS, IPAC, MPE, RAL and SRON.
} 

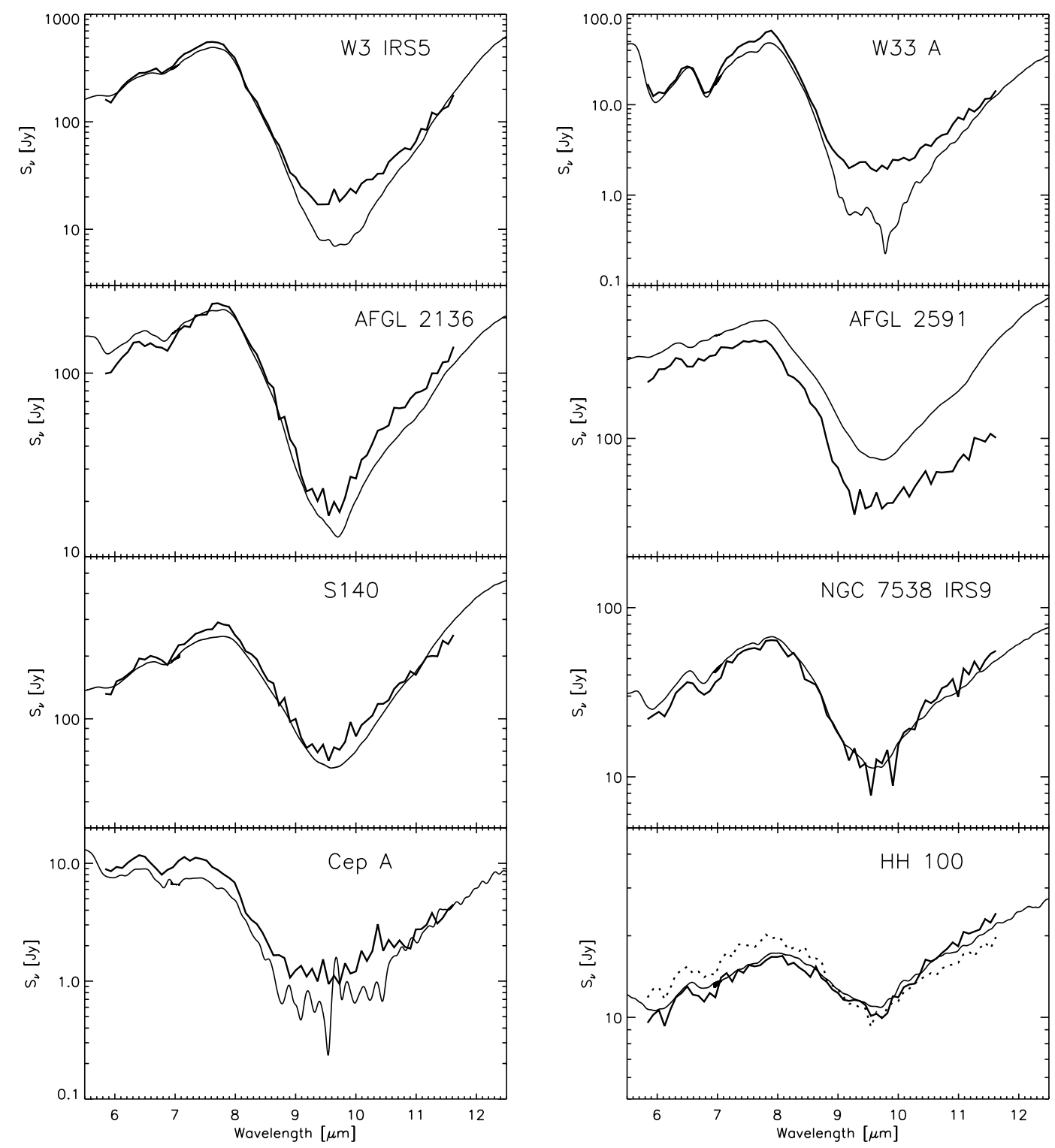

Fig. 1. Comparison of the PHT-SL spectra (thick solid line) with the SWS spectra (thin solid line). The SWS spectra are smoothed with a Gaussian filter with a width of $0.096 \mu \mathrm{m}$ to mimic the spectral resolution of PHT-S spectra. A second PHT-S observation of HH 100-IRS is shown by a dotted line. The low flux of the PHT-S spectrum of AFGL 2591 indicates a pointing off-set with respect to the SWS spectrum.

Lacy et al. (1998) suggest that the $\mathrm{NH}_{3}$ band extends from 8.5 to $9.5 \mu \mathrm{m}$ if these molecules are embedded in water ice. This spectral region has, therefore, to be excluded from the fitting procedure. The fitting was done to the logarithms of the flux values versus a logarithmic wavelength scale. Using the logarithms of the flux values in the fitting procedure takes advantage of a certain similarity of the underlying silicate profile without presuming a unique one. It cannot be expected to fit a large part of the silicate band by this method if we do not use polynomials of a very high order. However, these tend to lead to unstable and oscillating fits. Care is necessary with the choice of the wavelength intervals the fits are to be based on. After some testing, we chose $8.2-8.6$ and $9.4-9.8 \mu \mathrm{m}$ as the fitting intervals and a 3rd order polynomial for processing the PHT-S spectra and 8.4-8.6 and 9.4-9.6 $\mu \mathrm{m}$ and a 4 th order polynomial for processing the SWS spectra. The fact that the 8.6-9.4 $\mu \mathrm{m}$ range is well matched by the fits in the spectra of those sources for which we have independent indication that no ammonia feature is present provides confidence that our method is suitable for this kind of analysis.

The $\mathrm{NH}_{3}$ band region in the PHT-S and SWS spectra of our sources are shown in Figs. 2 and 3, respectively. The band is clearly seen in the PHT-S spectrum of W33A and may be present in 3 additional sources (AFGL 2136, Cep A, and Barnard 5). The SWS spectra confirm the results for W33A and AFGL 2136, but show the band also in HH 100-IRS and NGC 7538:IRS9. The Cep A spectrum is too noisy for giving convincing results. Central optical depths $\tau_{\mathrm{NH}_{3}}$ for the band were estimated and are given in Table 1.

The parameters of the extracted $\mathrm{NH}_{3}$ band depend on the order of the polynomial used and the range chosen to determine the baseline for the $\mathrm{NH}_{3}$ band. For illustrative purposes we use NGC 7538:IRS9 as an example. Strictly following the procedure of Lacy et al. (1998) would mean choosing 

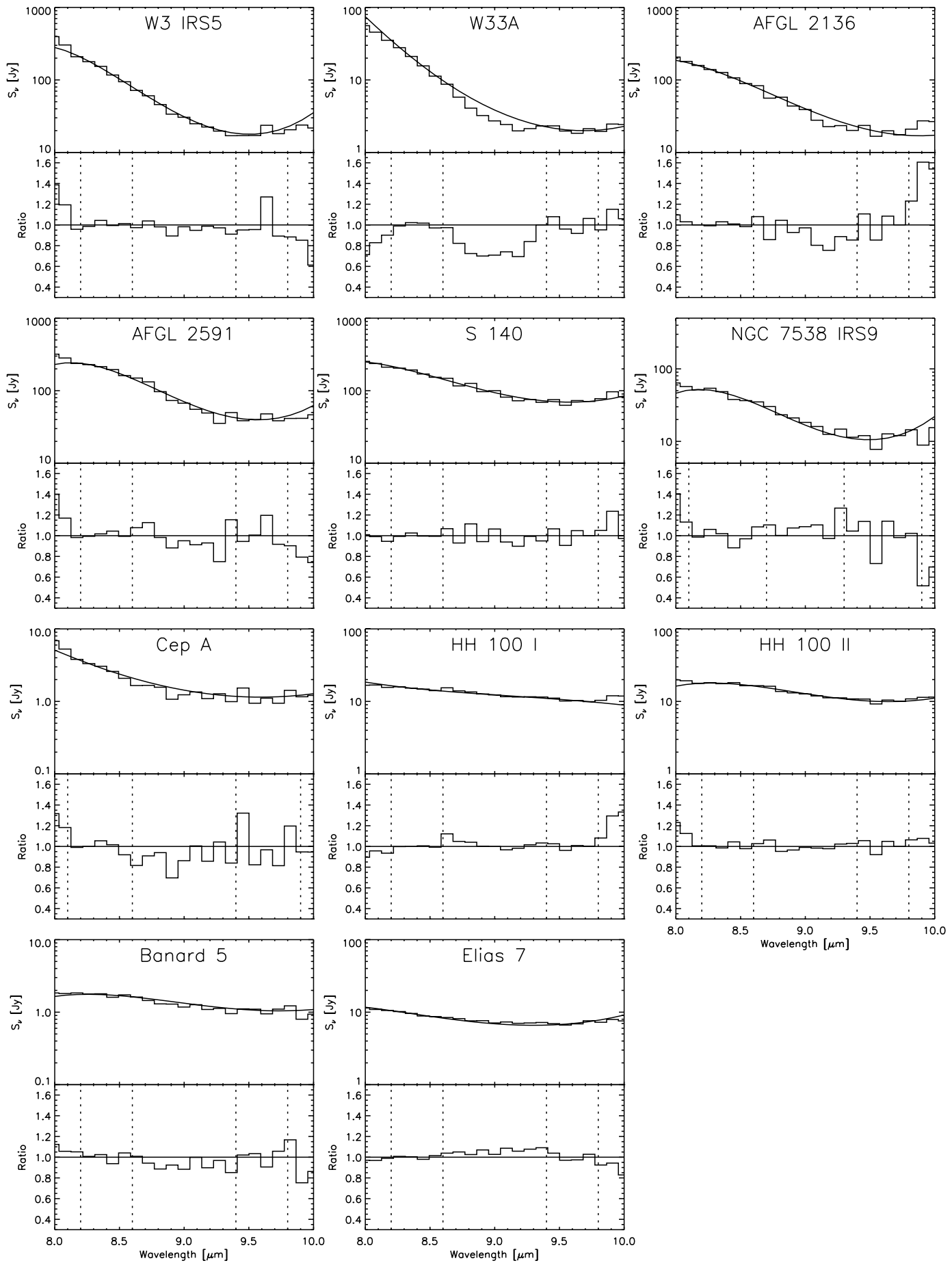

Fig. 2. Results of the analysis of PHT-S observations in the range around the $\mathrm{NH}_{3}$ inversion band at $9.0 \mu \mathrm{m}$. The upper panels show the PHT-SL spectra with the fitted polynomials. In the lower panels we plotted the ratio of the interpolated to the observed flux. The dashed vertical lines indicate the two fit ranges. (For details see the text.)

a polynomial of the first order and fitting it to the regions 8.48.6 $\mu \mathrm{m}$ and 9.4-9.6 $\mu \mathrm{m}$. Doing this, the PHT-S data do not reveal a feature. However, it has to be noted that due to the spectral resolution, either region includes 2 pixels of the PHT$\mathrm{S}$ array, which makes the baseline determination sensitive to measurement uncertainties of individual pixels. Indeed, omitting the deviating data point at $9.5 \mu \mathrm{m}$ results in an optical depth of $\tau_{\mathrm{NH}_{3}} \approx 0.1$. If we wish to include some more pixels on either side, we have to take the curvature of the silicate feature into account. Fitting a 2nd order polynomial to the regions 8.4-8.6 and 9.4-9.6 $\mu \mathrm{m}$ results in a feature of $\tau_{\mathrm{NH}_{3}}=0.15$. Extending the fitting regions to 8.2-8.6, 9.4-9.8 $\mu \mathrm{m}$ makes the feature disappear. Fitting a polynomial of 3rd order to the regions 8.2-8.6 and 9.4-9.8 $\mu \mathrm{m}$ produces an excess in the band region. Only broadening the interval $8.1-8.7$ and 9.3-9.9 $\mu \mathrm{m}$ 

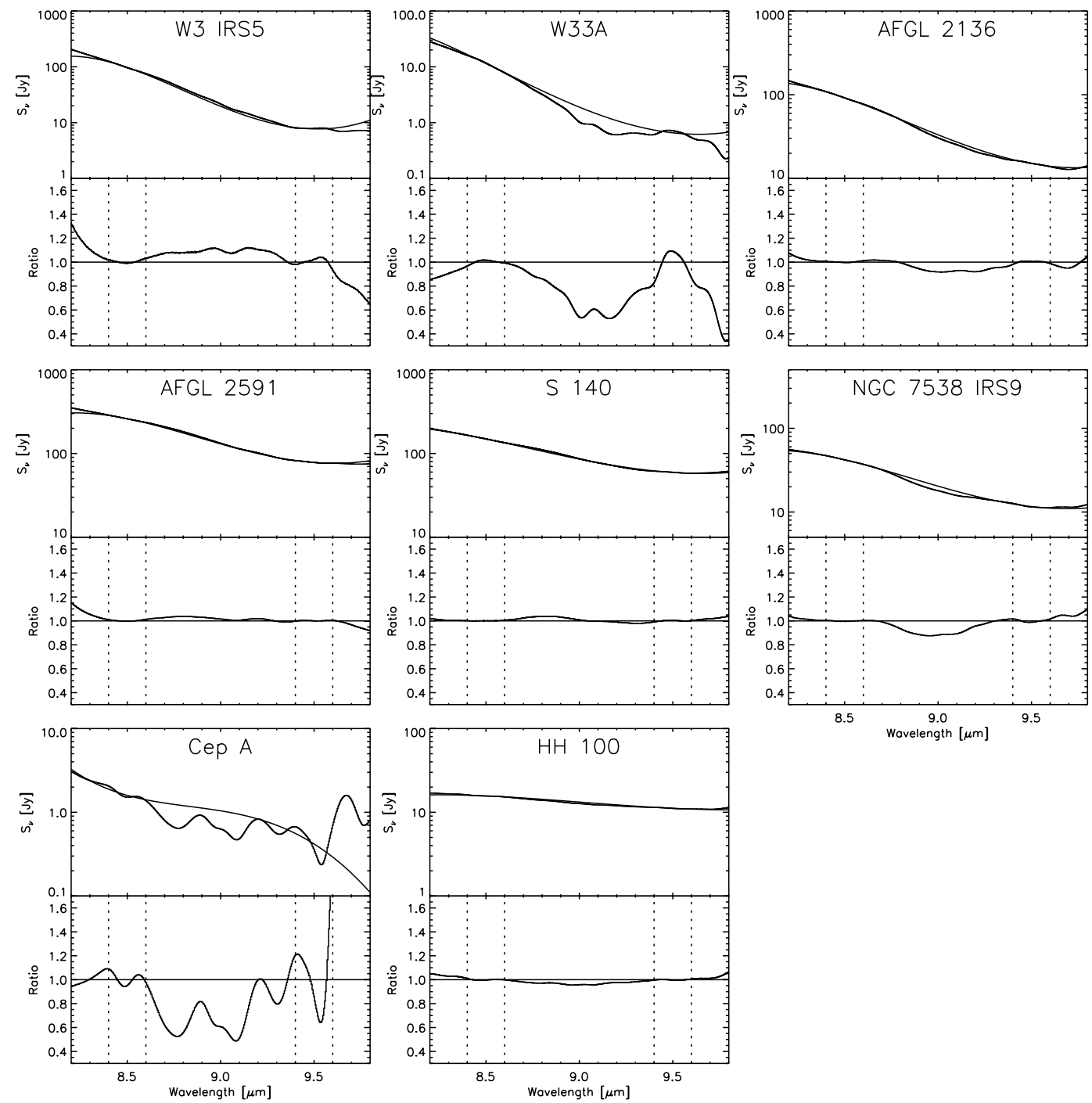

Fig. 3. Results of the analysis of the SWS observations in the range around the $\mathrm{NH}_{3}$ inversion band at $9.0 \mu \mathrm{m}$. Below a flux of $1 \mathrm{Jy}$ the spectra are relatively noisy and the smoothing produces wiggles, which are particularly pronounced in the spectrum of Cep A. The upper panels show the SWS spectra with the fitted polynomials. In the lower panels we plotted the ratio of the interpolated to the observed flux. The dashed vertical lines indicate the two fit ranges.

made the excess disappear. Choosing a polynomial of 4th or the 5 th order gave virtually the same result.

We estimate from such tests that the uncertainty of the optical depth $\tau_{\mathrm{NH}_{3}}$ derived from the PHT-S spectra is \pm 0.1 .

For NGC 7538:IRS9 Lacy et al. (1998) found $\tau_{\mathrm{NH}_{3}} \approx 0.31$, while we see no feature in the PHT-S spectra. On the other hand, we derived an optical depth for the $\mathrm{NH}_{3}$ band in W33A that is remarkably similar to the one given by Gibb et al. (2000).

We used the same procedure for a search for the $\mathrm{CH}_{3} \mathrm{OH}$ band at $9.7 \mu \mathrm{m}$. We found this band in 5 sources. The spectra are shown in Figs. 4 and 5, respectively. The derived optical depths are listed in Table 1. Their uncertainty is of the order of \pm 0.1 .

The procedure was also used to search for the band of solid $\mathrm{CH}_{4}$ at $7.7 \mu \mathrm{m}$ in the PHT-S spectra. Here the fit range was $7.3-7.6$ and $7.8-8.1 \mu \mathrm{m}$. The band was detected in 5 sources, in two of them (Barnard 5 and HH 100-IRS) only tentatively. The spectra are displayed in Fig. 6. The results were again compared with the SWS spectra, which we display in Fig. 7. Because of the smoother spectra and the generally higher flux levels, the uncertainty of the optical depths is estimated at \pm 0.05 .

\section{Discussion}

Laboratory work on interstellar ice analogues predicts the broad $10 \mu \mathrm{m}$ band to be overlaid by the $\mathrm{NH}_{3}$ band around $9.0 \mu \mathrm{m}$ and the $\mathrm{CH}_{3} \mathrm{OH}$ band at $9.7 \mu \mathrm{m}$ (d'Hendecourt \& Allamandola 1986). In addition, the signature of $\mathrm{CH}_{4}$ ice is expected at $7.7 \mu \mathrm{m}$.

Our analysis of the PHT-S spectra presents clear evidence of the $\mathrm{NH}_{3}$ band in one and traces of its presence in another 3 out of 10 sources investigated. Evidence of the $\mathrm{CH}_{3} \mathrm{OH}$ band was found in 5 sources and of the $\mathrm{CH}_{4}$ band in 5 sources, too. In Table 2 we give column densities of these three ice components. The values were derived by using the integrated 
Table 1. Optical depths of the $9.0 \mu \mathrm{m}$ ammonia, $9.7 \mu \mathrm{m}$ methanol, and $7.7 \mu \mathrm{m}$ methane ice bands. Bad data which did not allow that meaningful values were derived are marked by an asterisk. For more details see the text.

\begin{tabular}{llcccccc}
\hline \hline \multirow{2}{*}{$\begin{array}{l}\text { IRAS } \\
\text { name }\end{array}$} & other & \multicolumn{2}{c}{$\tau_{\mathrm{NH}_{3}}$} & \multicolumn{2}{c}{$\tau_{\mathrm{CH}_{3} \mathrm{OH}}$} & \multicolumn{2}{c}{$\tau_{\mathrm{CH}_{4}}$} \\
& name & PHT-S & SWS & PHT-S & SWS & PHT-S & SWS \\
\hline $02219+6152$ & W3:IRS5 & $<0.1$ & $<0.05$ & $<0.1$ & $<0.1$ & $<0.05$ & 0.02 \\
$03445+3242$ & Barnard 5 & 0.1 & - & $<0.1$ & - & 0.04 & - \\
$04263+2426$ & Elias 7=Haro 6-10 & $<0.1$ & - & $<0.1$ & - & $<0.05$ & - \\
$18117-1753$ & W33A & 0.3 & 0.56 & 0.25 & 0.62 & 0.1 & 0.11 \\
$18196-1331$ & AFGL 2136 & 0.1 & 0.07 & 0.25 & 0.22 & $<0.05$ & 0.03 \\
& HH 100 (I) & $<0.1$ & 0.04 & 0.15 & $<0.05$ & 0.03 & 0.02 \\
& HH 100 (II) & $<0.1$ & - & 0.1 & - & 0.02 & - \\
$20275+4001$ & AFGL 2591 & $*$ & $<0.04$ & $*$ & $<0.05$ & $*$ & 0.02 \\
$22176+6303$ & S140:IRS1 & $<0.1$ & $<0.04$ & 0.2 & $<0.05$ & $<0.05$ & $<0.01$ \\
$22543+6145$ & Cep A & 0.1 & $*$ & 0.2 & $*$ & 0.05 & 0.07 \\
$23118+6110$ & NGC 7538:IRS9 & $<0.1$ & 0.15 & $<0.1$ & 0.07 & 0.1 & 0.05 \\
\hline
\end{tabular}

Table 2. Derived column densities of ammonia, methanol, and methane ice components.

\begin{tabular}{llcccccc}
\hline \hline $\begin{array}{l}\text { IRAS } \\
\text { name }\end{array}$ & $\begin{array}{l}\text { other } \\
\text { name }\end{array}$ & \multicolumn{2}{c}{$N\left(\mathrm{NH}_{3}\right)$} & \multicolumn{2}{c}{$N\left(\mathrm{CH}_{3} \mathrm{OH}\right)$} & \multicolumn{2}{c}{$N\left(\mathrm{CH}_{4}\right)$} \\
& & $\begin{array}{c}{\left[10^{17} \mathrm{~cm}^{-2}\right]} \\
\text { PHT-S }\end{array}$ & SWS & \multicolumn{2}{c}{$\left[10^{17} \mathrm{~cm}^{-2}\right]$} & \multicolumn{2}{c}{$\left[10^{17} \mathrm{~cm}^{-2}\right]$} \\
\hline $02219+6152$ & W3:IRS5 & $<4$ & $<1.9$ & $<2$ & $<0.2$ & $<0.6$ & 0.23 \\
$03445+3242$ & Barnard 5 & 4 & - & $<2$ & - & 0.5 & - \\
$04263+2426$ & Elias 7=Haro 6-10 & $<4$ & - & $<2$ & - & $<0.6$ & - \\
$18117-1753$ & W33A & 12 & 22 & 5 & 13 & 1 & 1.3 \\
$18196-1331$ & AFGL 2136 & 4 & 2.7 & 5 & 4.6 & $<0.6$ & 0.34 \\
& HH 100 & $<4$ & 1.5 & 3 & $<0.1$ & 0.3 & 0.22 \\
$20275+4001$ & AFGL 2591 & $*$ & $<1.5$ & $*$ & $<0.1$ & $*$ & 0.22 \\
$22176+6303$ & S140:IRS1 & $<4$ & $<1.5$ & 4 & $<0.1$ & $<0.6$ & $<0.1$ \\
$22543+6145$ & Cep A & 4 & $*$ & 4 & $*$ & 0.6 & 0.80 \\
$23118+6110$ & NGC 7538:IRS9 & $<4$ & 6 & $<2$ & 1.4 & 1 & 0.57 \\
\hline
\end{tabular}

Table 3. Optical depths and/or column densities of gaseous ammonia, silicate dust, and various ice components.

\begin{tabular}{llccccccl}
\hline \hline $\begin{array}{l}\text { IRAS } \\
\text { name }\end{array}$ & $\begin{array}{l}\text { other } \\
\text { name }\end{array}$ & $\begin{array}{c}N_{\mathrm{g}}\left(\mathrm{NH}_{3}\right) \\
{\left[10^{14} \mathrm{~cm}^{-2}\right]}\end{array}$ & $\tau_{\text {sil }}$ & $\tau_{\mathrm{H}_{2} \mathrm{O}}$ & $\begin{array}{c}N\left(\mathrm{H}_{2} \mathrm{O}\right) \\
{\left[10^{17} \mathrm{~cm}^{-2}\right]}\end{array}$ & $\frac{N(\mathrm{CO})}{N\left(\mathrm{H}_{2} \mathrm{O}\right)}$ & $\frac{N\left(\mathrm{CO}_{2}\right)}{N\left(\mathrm{H}_{2} \mathrm{O}\right)}$ & Ref. \\
\hline $02219+6152$ & W3:IRS5 & 0.3 & 7.64 & 3.00 & 54 & 0.030 & 0.13 & $(1),(2),(3)$ \\
$03445+3242$ & Barnard 5 & $<3.2$ & $1:$ & 1.2 & 23 & - & - & $(1),(4)$ \\
$04263+2426$ & Elias 7=Haro 6-10 & 2 & $1:$ & 0.5 & 10 & - & - & $(5)$ \\
$18117-1753$ & W33A & 10 & 7.84 & 5.5 & 110 & 0.10 & 0.13 & $(6),(2),(7),(3)$ \\
$18196-1331$ & AFGL 2136 & - & 5.07 & 3.2 & 50 & 0.022 & 0.16 & $(2),(3)$ \\
& HH 100 (I) & - & 1.21 & 1.44 & 27 & - & - & $(9)$ \\
$20275+4001$ & AFGL 2591 & 1.6 & 4.14 & 0.71 & 17 & $<0.012$ & 0.094 & $(1),(2),(8),(3)$ \\
$22176+6303$ & S140:IRS1 & 7.9 & 3.97 & 1.23 & 21.5 & $<0.010$ & 0.20 & $(1),(2),(8),(3)$ \\
$22543+6145$ & Cep A & 3.8 & $7.5:$ & 2.6 & 40 & - & - & $(10)$ \\
$23118+6110$ & NGC 7538:IRS9 & 1.2 & 4.46 & 3.28 & 75 & 0.15 & 0.20 & $(10),(2),(3)$ \\
\hline
\end{tabular}

References: (1) Jijima et al. (1999); (2) Willner et al. (1982); (3) Gerakines et al. (1999); (4) Charley et al. (1990); (5) Anglada et al. (1989); (6) Mauersberger et al. (1985); (7) Gibb et al. (2000) (8) Dartois \& d'Hendecourt (2001) (9) Whittet et al. (1996); (10) Churchwell et al. (1990).

absorbances and full widths at half maximum measured by Kerkhof et al. (1999) and d'Hendecourt \& Allamandola (1986), respectively. In Fig. 8 we compare the profile of the extracted $\mathrm{NH}_{3}$ bands with the laboratory spectrum given by Lacy et al. (1998). Excess absorption seems to be present on the long-wavelength side of the band. Similar additional absorption (a "9.2 $\mu \mathrm{m}$ feature") was already noted by Gibb et al. (2000, 2001) and tentatively attributed to a crystalline or semicrystalline Mg-rich silicate component.

In the following, we will discuss the sources individually. 

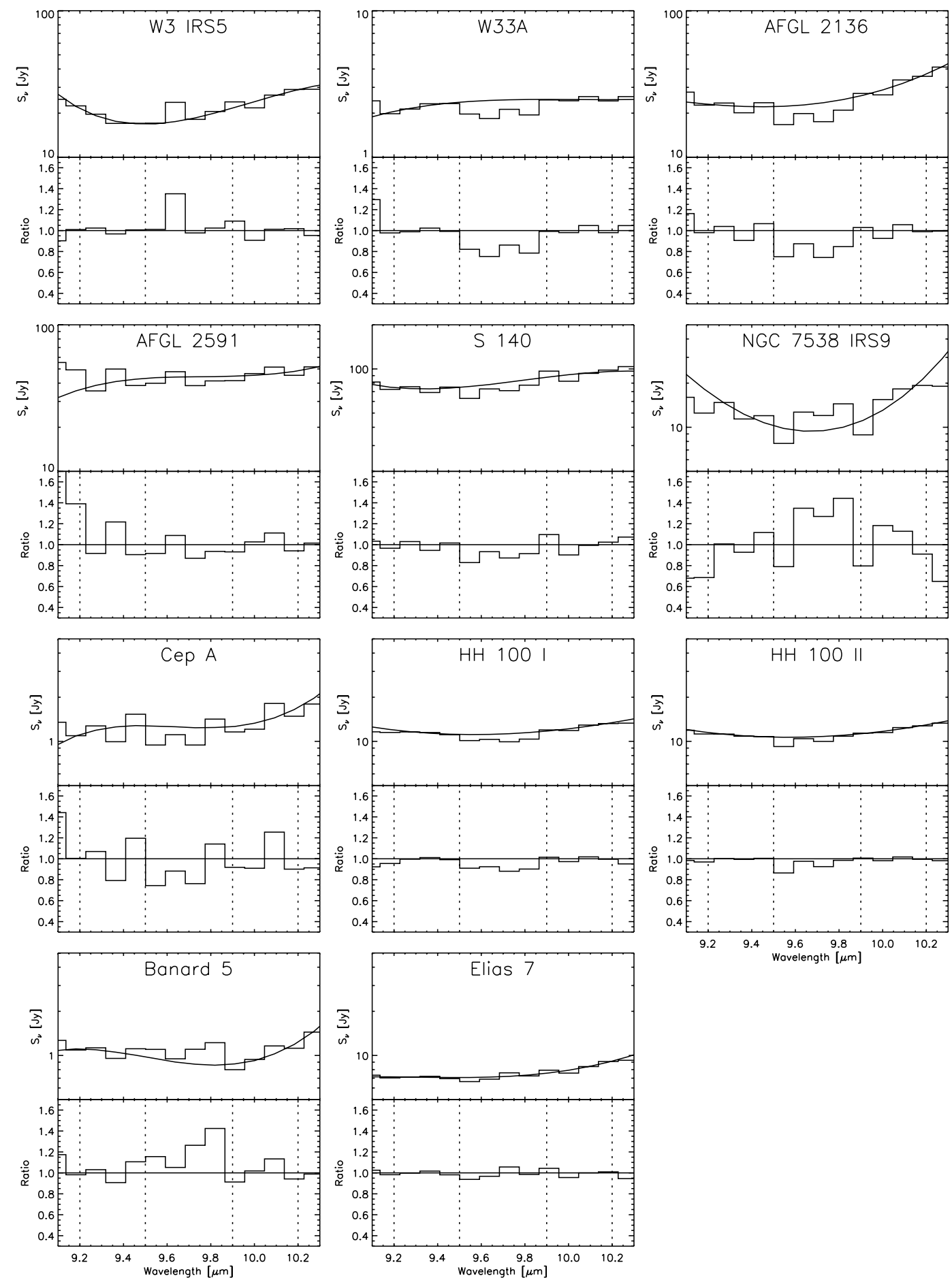

Fig. 4. Results of the analysis of PHT-S observations in the range around the $\mathrm{CH}_{3} \mathrm{OH}$ band at $9.7 \mu \mathrm{m}$. The upper panels show the PHT-SL spectra with the fitted polynomials. In the lower panels we plotted the ratio of the interpolated to the observed flux. The dashed vertical lines indicate the two fit ranges.

W33A: all three bands we searched for were found in the PHT-S spectrum and their presence was confirmed by the SWS spectrum. As can be seen from Fig. 8, the profile of the $\mathrm{NH}_{3}$ band is in excellent agreement with the laboratory spectrum reported by Lacy et al. (1998). From the PHT-S data we derived significantly smaller optical depths than from the SWS data. Our SWS result is consistent with the result of Gibb et al. (2000) who found $\tau_{\mathrm{NH}_{3}}=0.45 \pm 0.10$ from their analysis of the SWS spectrum. One important reason for these differences may be the different sizes of the field of view of the two instruments. The entrance area of the SWS instrument is $280 \square^{\prime \prime}$, the entrance area of PHT-S $576 \square^{\prime \prime}$. As can be seen in Fig. 1, PHT-S measured a higher flux than SWS all over the spectrum, with the largest differences within the features. For instance, SWS has measured $1 \mathrm{Jy}$ at the minimum of the silicate band, whereas the PHT spectrum gives 2.3 Jy. The simplest 

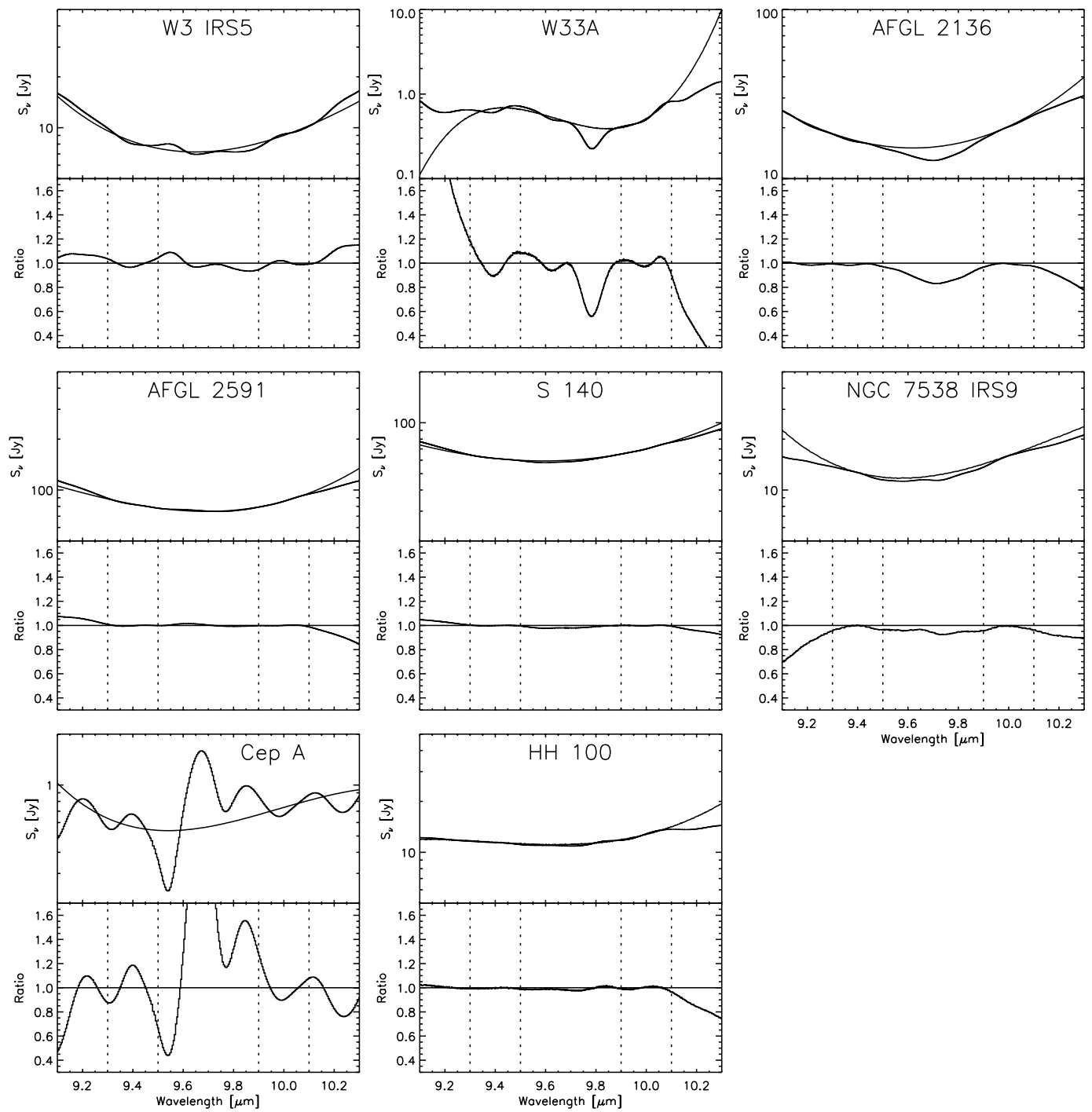

Fig. 5. Results of the analysis of the SWS observations in the range around the $\mathrm{CH}_{3} \mathrm{OH}$ band at $9.7 \mu \mathrm{m}$. Below a flux of $1 \mathrm{Jy}$ the spectra are relatively noisy and the smoothing produces wiggles, which are particularly pronounced in the spectrum of Cep A. The upper panels show the SWS spectra with the fitted polynomials. In the lower panels we plotted the ratio of the interpolated to the observed flux. The dashed vertical lines indicate the two fit ranges.

explanation of these differences is that the source is extended and the bands are markedly weaker in the additional flux received by PHT-S. It is noteworthy that Lacy et al. (1998) were unable to detect this band in their ground-based spectrum.

Within our sample, W33A is the source with both the deepest $9.0 \mu \mathrm{m}$ band and the deepest $3.08 \mu \mathrm{m} \mathrm{H}_{2} \mathrm{O}$ band. Adopting an integrated absorbance of $A=1.3 \times 10^{-17} \mathrm{~cm} \mathrm{molecule}^{-1}$ and a full width at half maximum of $\Delta v_{1 / 2}=50 \mathrm{~cm}^{-1}$ (d'Hendecourt \& Allamandola 1986; Kerkhof et al. 1999), an optical depth $\tau_{\mathrm{NH}_{3}}=0.5$, and an $\mathrm{H}_{2} \mathrm{O}$ ice column density of $1.1 \times 10^{19} \mathrm{~cm}^{-2}$ (Gibb et al. 2000) results in an $N\left(\mathrm{NH}_{3}\right) / N\left(\mathrm{H}_{2} \mathrm{O}\right)$ ratio of 0.2 . Laboratory spectra of $\mathrm{H}_{2} \mathrm{O}: \mathrm{NH}_{3}=1: 0.25$ mixtures show the depth of the $3.08 \mu \mathrm{m} \mathrm{H}_{2} \mathrm{O}$ and $2.95 \mu \mathrm{m} \mathrm{NH}$ bands at the same level. The PHT-S data cannot be used to separate these bands. However, a comparison of the $3 \mu \mathrm{m}$ features of W33A and Cep A (for which our data suggest a $N\left(\mathrm{NH}_{3}\right) / N\left(\mathrm{H}_{2} \mathrm{O}\right)$ ratio of 0.1 only) points to the presence of additional absorption at the short-wavelength wing in the case of W33A (cf. Fig. 9). Thus, the shape of the $3 \mu \mathrm{m}$ feature in W33A is qualitatively compatible with the $\mathrm{NH}_{3} / \mathrm{H}_{2} \mathrm{O}$ abundance ratio derived from the $9.0 \mu \mathrm{m}$ band. It is worth mentioning that the depth of the $\mathrm{H}_{2} \mathrm{O}$ ice band is affected by the different fields of view of the PHT-S and SWS instruments in a similar way as the depth of the silicate feature. Consequently its central optical depth is lower by a factor of about two in the PHT-S spectra. While the optical depth of the superposed $\mathrm{NH}_{3}$ stretching band also decreases, the effect does not wipe out the band from the underlying $\mathrm{H}_{2} \mathrm{O}$ feature.

The $\mathrm{CH}_{3} \mathrm{OH}$ abundance from our PHT-S and SWS data is smaller than that derived by Dartois et al. (1999) from the $3.54 \mu \mathrm{m}$ band.

The $\mathrm{CH}_{4}$ band at $7.7 \mu \mathrm{m}$ is clearly present in our spectrum. The optical depth agrees favourably with the result of Boogert et al. $(1996,1998)$.

W3:IRS5: none of the bands were found in the PHT-S spectra. This is in accordance with the SWS data, which show only a very weak $\mathrm{CH}_{4}$ band with an maximum optical depth $\tau_{\mathrm{CH}_{4}} \approx 0.02$. In spite of showing one of the deepest silicate 

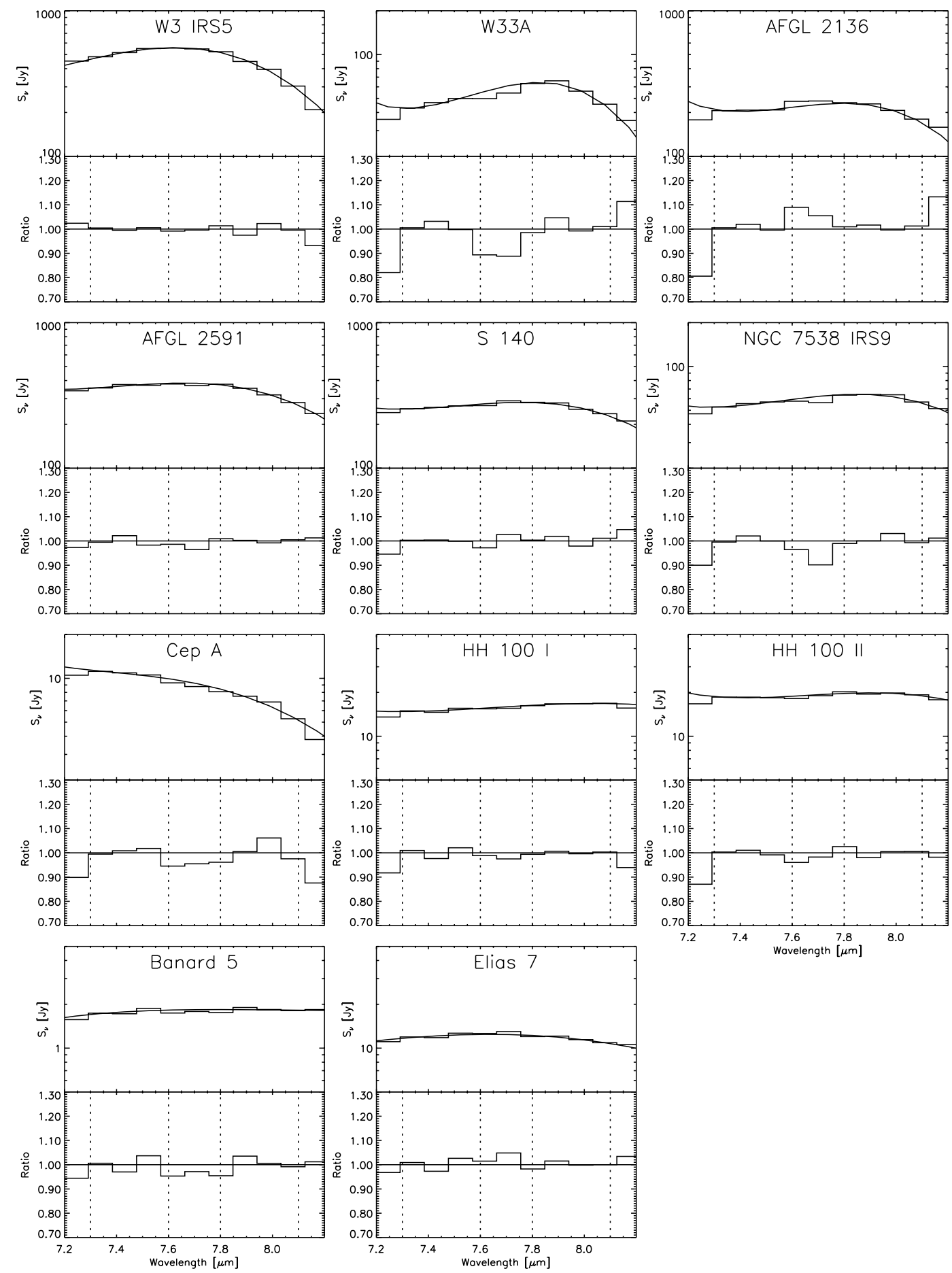

Fig. 6. Results of the analysis of PHT-S observations in the range around the $\mathrm{CH}_{4}$ band at $7.7 \mu \mathrm{m}$. The upper panels show the PHT-SL spectra with the fitted polynomials. In the lower panels we plotted the ratio of the interpolated to the observed flux. The dashed vertical lines indicate the two fit ranges. The dashed lines indicate the fit range from 7.3 to 7.6 and 7.8 to $8.1 \mu \mathrm{m}$.

bands of our sample, there is no indication of the " $9.2 \mu \mathrm{m}$ feature". This fact was also mentioned by Gibb et al. (2001) and argues against a simple correlation between this feature and the amount of silicate dust along the line of sight.

If the PHT-S data are compared to the SWS data, we find a favourable agreement of the flux levels shortward of $8.5 \mu \mathrm{m}$, while at the centre of the silicate band the PHT-S flux is higher by a factor of $\approx 2.7$. We attribute most of this difference to the different fields of view of the instruments. The infrared maps presented by Hackwell et al. (1978) show that W3:IRS5 is extended at mid-IR wavelengths and that especially when observing at the centre of the silicate band, PHT-S is expected to receive additional flux. 

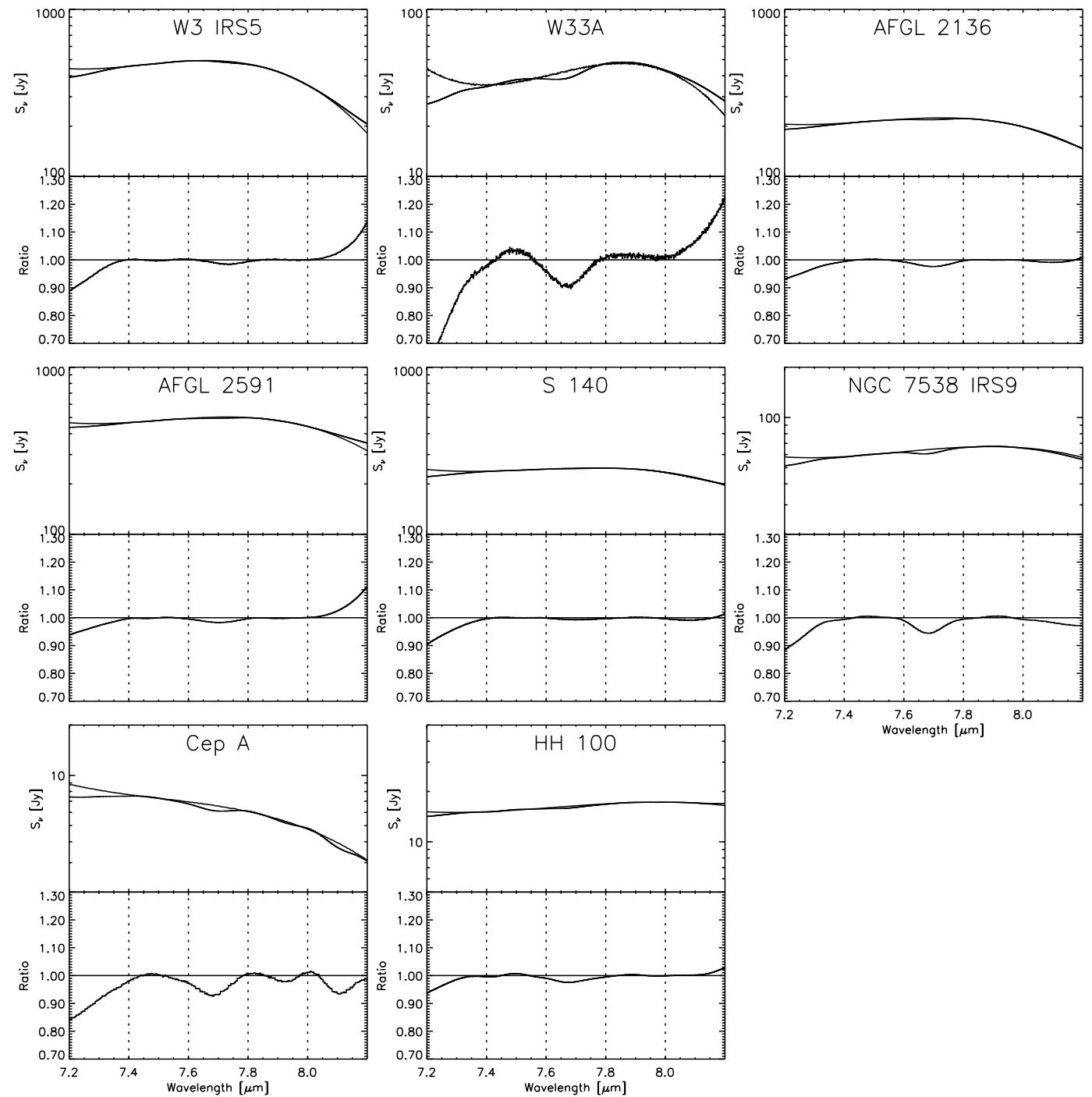

Fig. 7. Results of the analysis of the SWS observations in the range around the $\mathrm{CH}_{4}$ band at $7.7 \mu \mathrm{m}$. Below a flux of $1 \mathrm{Jy}$ the spectra are relatively noisy and the smoothing produces wiggles, which are particularly pronounced in the spectrum of Cep A. The upper panels show the SWS spectra with the fitted polynomials. In the lower panels we plotted the ratio of the interpolated to the observed flux. The dashed vertical lines indicate the two fit ranges.

Barnard 5: the profile of the weak $\mathrm{NH}_{3}$ band agrees well with the laboratory spectrum (Fig. 8). Its depth implies an $N\left(\mathrm{NH}_{3}\right) / N\left(\mathrm{H}_{2} \mathrm{O}\right)$ ratio of 0.07 .

$A F G L$ 2136: the data from PHT-S and SWS agree very well between 7.5 and $12 \mu \mathrm{m}$, which is the wavelength interval we are most interested in. The $\mathrm{NH}_{3}$ band is very weak in both spectra, but of a comparable depth. The observed PHT-S profile is not fitted well by the laboratory spectrum (cf. Fig. 8). It seems to be dominated by excess absorption around $9.2 \mu \mathrm{m}$. Our SWS spectrum shows this excess absorption, but to a lesser degree. The optical depth $\tau_{\mathrm{NH}_{3}}$ estimated from the PHT-S spectrum is very uncertain, but we found a similar depth from the SWS spectrum. Gibb et al. (2001) derived an upper limit of $\tau_{\mathrm{NH}_{3}} \leq$ 0.02 , but their fit of the silicate profile is not fully convincing.

From ground-based spectroscopy Skinner et al. (1992) detected the $\mathrm{CH}_{3} \mathrm{OH}$ band with an optical depth of 0.25 . Our spectra are compatible with this result. Skinner et al. (1992) did not search for the $\mathrm{NH}_{3}$ band. Their silicate profile shows a shoulder at $9.0 \mu \mathrm{m}$ which may be interpreted as an indication of the $\mathrm{NH}_{3}$ band. Its depth is compatible with our results.

The $\mathrm{CH}_{4}$ band is absent in the PHT-S data. In the SWS spectrum the $\mathrm{CH}_{3} \mathrm{OH}$ band is very distinct with an optical depth of $\approx 0.2$, whereas the $\mathrm{CH}_{4}$ band is very weak.

HH 100-IRS: because both independent PHT-S spectra indicate the presence of the $\mathrm{CH}_{3} \mathrm{OH}$ band, the feature is probably real. The methanol abundance derived from it agrees with the upper limit by Dartois et al. (1999).

AFGL 2591: the flux level of the SWS spectrum is significantly higher than the flux level of the PHT-S spectrum. The cause of it is that the positions used in the respective observations differ by $\approx 11^{\prime \prime}$. This off-set places the source at the very edge of the PHT-S aperture, where the relative response correction does not compensate for the signal loss on the wing of the beam profile. The weak $\mathrm{NH}_{3}$ band is probably an artefact. It is not seen in the SWS spectrum. A weak $\mathrm{CH}_{4}$ band of comparable strength is seen in both spectra. 

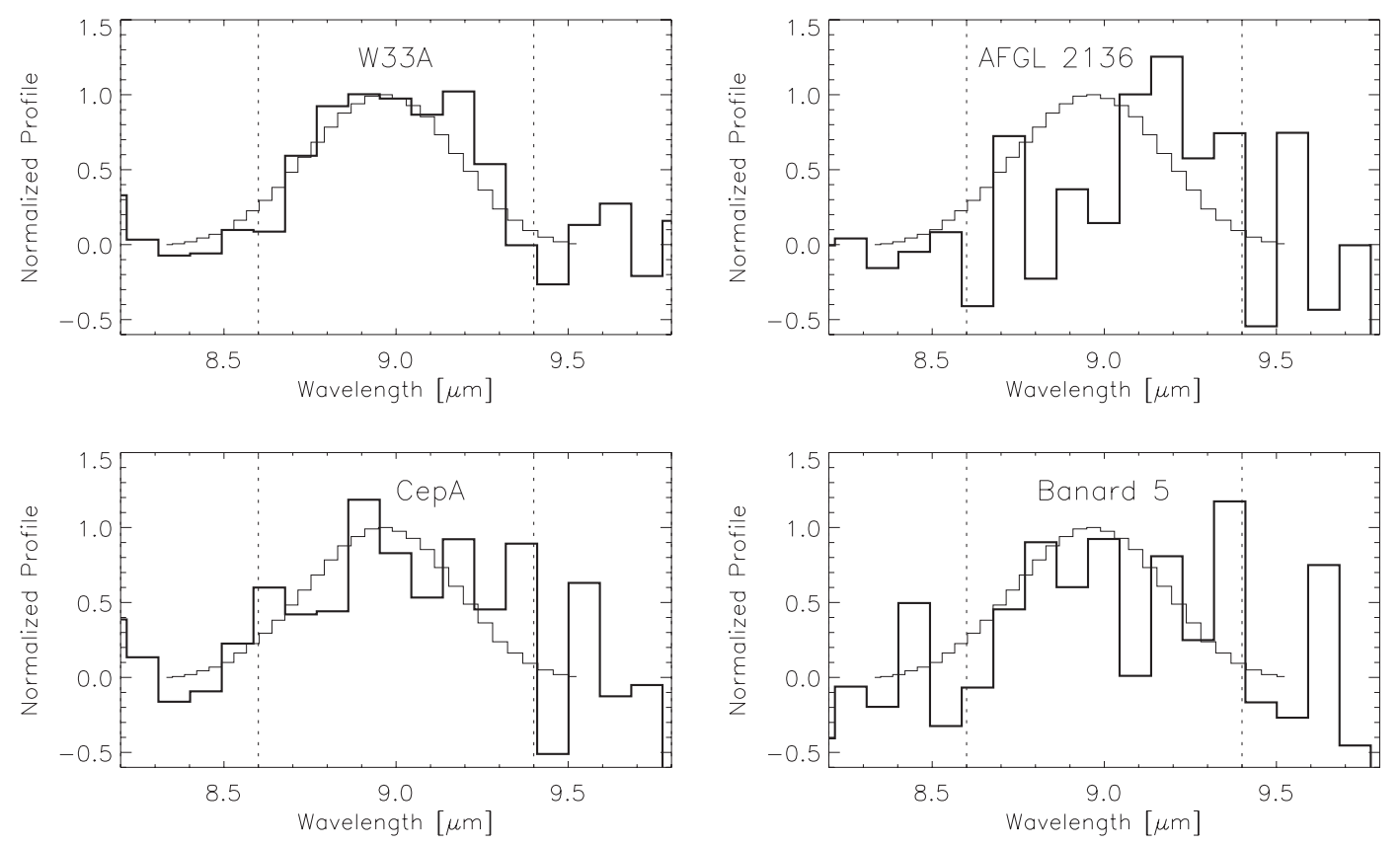

Fig. 8. Comparison of the profiles of the $\mathrm{NH}_{3}$ inversion band from the PHT-S spectra (thick line) with the laboratory data (thin line) of Lacy et al. (1998). The PHT-S profiles are normalized by using the three lowest points within the band range, which is indicated by the dashed vertical lines.
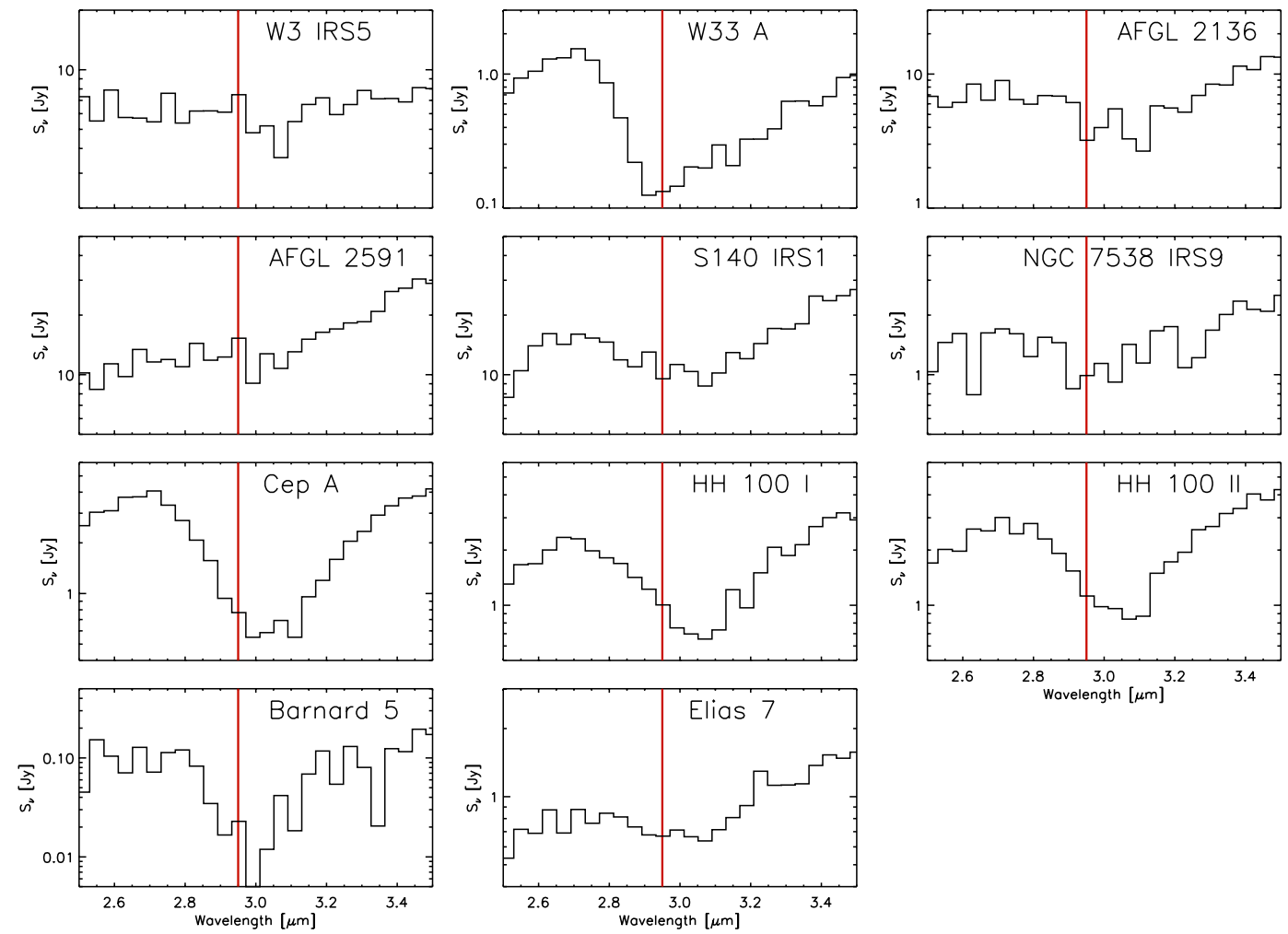

Fig. 9. PHT-S observations of the region of the $\mathrm{H}_{2} \mathrm{O}$ ice band at $3.08 \mu \mathrm{m}$. The vertical line indicates the position of the $\mathrm{NH}_{3}$ stretching band.

S140:IRS1: both spectra agree well. There is an indication of the methanol feature in the PHT-S spectrum whereas the SWS spectrum shows a minimal dip at best. If this feature is real, it implies a larger column density than the upper limit derived by Dartois et al. (1999) from the $3.54 \mu \mathrm{m}$ band.
NGC 7538:IRS9: no $\mathrm{NH}_{3}$ band was detected in our PHT-S spectrum. This contradicts the results of Lacy et al. (1998), who found $\tau_{\mathrm{NH}_{3}}=0.31$. As already discussed in Sect. 3, one reason is that the spectrum grows more noisy beyond $9.0 \mu \mathrm{m}$, which makes the polynomial fit very sensitive to the 
intervals the fit is based on. On the other hand, the SWS spectrum agrees excellently with the PHT-S spectrum. The depth of the $\mathrm{NH}_{3}$ band is estimated to be $\approx 0.15$. Gibb et al. (2001) give $\tau_{\mathrm{NH}_{3}}=0.20 \pm 0.05$ from their analysis of the SWS data. Thus, it seems that the strength of this band was overestimated from the ground-based data, possibly by an inappropriate approximation of the silicate profile.

No methanol band is seen in the PHT-S spectrum. The weak feature in the SWS spectrum implies a significantly smaller methanol abundance than that derived by Allamandola et al. (1992) from the $3.54 \mu \mathrm{m}$ band.

The $\mathrm{CH}_{4}$ band is clearly present. The $\mathrm{CH}_{4}$ feature seen in the SWS spectrum was already discussed by Boogert et al. (1996, 1998).

Cep A: the PHT-S and SWS spectra agree very well. However, the SWS spectrum is very noisy beyond $8.5 \mu \mathrm{m}$. Cep A shows a silicate band, whose depth is comparable with that in W3:IRS5 and W33 A. The $\mathrm{H}_{2} \mathrm{O}$ ice band is only about half as deep as that in W33 A is. At the bottom of the silicate band the noise in the PHT-S spectrum is relatively large. Therefore, the polynomial fit and, consequently, the optical depth of the $\mathrm{NH}_{3}$ band are particularly uncertain. The observed profile agrees well with the laboratory spectrum (Fig. 8). The small optical depth of the $\mathrm{NH}_{3}$ band goes well with the profile of the $3.08 \mu \mathrm{m}$ $\mathrm{H}_{2} \mathrm{O}$ ice band. We have already mentioned that the shape of that band does not seem to be influenced by the presence of $\mathrm{NH}_{3}$ ice (see Fig. 9) pointing to a clearly smaller $\mathrm{NH}_{3} / \mathrm{H}_{2} \mathrm{O}$ ratio than in W33 A. The SWS spectrum does not allow to confirm this result. The $\mathrm{CH}_{3} \mathrm{OH}$ band seems to be present in the PHT-S spectrum. The $\mathrm{CH}_{4}$ band is seen in both spectra with comparable strength.

In Table 3 we have compiled some data on the silicate and various ice components present along the lines of sight to our sources. Moreover, we have listed the column densities of gaseous ammonia $N_{\mathrm{g}}\left(\mathrm{NH}_{3}\right)$.

Our results suggest that the abundance of $\mathrm{NH}_{3}$ ice can be comparable to that of $\mathrm{CO}_{2}$, i.e. $10-20 \%$ relative to water ice, in some cases. This number does not agree with the upper limit derived by Dartois \& d'Hendecourt (2001) on the base of their interpretation of the $3.47 \mu \mathrm{m}$ feature and lends support to the objections by Gibb et al. (2001). However, our results agree with the upper limits derived by Dartois \& d'Hendecourt (2001) from their modelling of the $3.08 \mu \mathrm{m} \mathrm{H}_{2} \mathrm{O}$ band.

The methanol ice abundances we have derived from the $9.7 \mu \mathrm{m}$ band are in most cases smaller than those that were reported from studies of the $3.54 \mu \mathrm{m}$ band. The maximum abundances are about $10 \%$ relative to water ice.

The abundance of methane ice is always small, amounting to $1-2 \%$ relative to water ice at best.

Our sample is evidently too small and the estimated optical depths too uncertain to establish correlations among the various ice components or with physical conditions. It is only obvious that neither a deep silicate feature (signalling much dust) nor a deep $\mathrm{H}_{2} \mathrm{O}$ band is sufficient for the presence of the $\mathrm{NH}_{3}$ ice feature. Different from solid $\mathrm{CO}$ and $\mathrm{CO}_{2}$, the abundance of $\mathrm{NH}_{3}$ ice is still elusive.

\section{Conclusions}

We searched mid-infrared spectra of 10 deeply embedded stellar objects for indications of the $9.0 \mu \mathrm{m}$ inversion band of solid $\mathrm{NH}_{3}$, the $9.7 \mu \mathrm{m}$ band of solid $\mathrm{CH}_{3} \mathrm{OH}$, and the $7.7 \mu \mathrm{m}$ band of solid $\mathrm{CH}_{4}$ in spectra taken with the spectrophotometer PHT$\mathrm{S}$ aboard the Infrared Space Observatory (ISO). The results were compared with spectra taken with the Short-Wavelength Spectrophotometer (SWS) of the same satellite.

We have detected the $\mathrm{NH}_{3}$ band in W33A and tentatively in Barnard 5, AFGL 2136, and Cep A. The SWS data confirm the results for W33A and AFGL 2136 and reveal the band in HH 100-IRS and NGC 7538:IRS9.

The $\mathrm{CH}_{3} \mathrm{OH}$ band was found in W33A, AFGL 2136, HH 100-IRS, S140:IRS1, and Cep A. It was confirmed by the SWS data for the first three sources and also seen in NGC 7538:IRS9.

The search for the $7.7 \mu \mathrm{m}$ band of $\mathrm{CH}_{4}$ resulted in five detections (Barnard 5, W33A, HH 100-IRS, Cep A, NGC 7538:IRS9). The SWS data confirm all the detections and add W3:IRS5 and AFGL 2591.

The good correspondence of the PHT-S and SWS findings for relatively bright sources demonstrates the applicability of our method and the complementary capabilities of the instruments SWS (high spectral resolution) and PHT-S (high sensitivity) on the Infrared Space Observatory.

Acknowledgements. The development and operation of ISOPHOT were supported by MPIA and funds from Deutsches Zentrum für Luft- und Raumfahrt e. V. (DLR, formerly DARA), with significant contributions to ISOPHOT-S from IAC, Tenerife, Spain. The ISOPHOT Data Centre at MPIA is supported by DLR with funds of Bundesministerium für Bildung und Forschung, grant no. 50 QI 9801 3. Use was made of the SIMBAD database, operated at CDS, Strasbourg, France. We thank Dr. E. Sturm from MPE Garching for his tutorial in SWS01 data reduction. We are grateful to the anonymous referee for his comments that helped to improve this study.

\section{References}

Allamandola, L. J., Sandford, S. A., Tielens, A. G. G. M., \& Herbst, T. M. 1992, ApJ, 399, 134

Anglada, G., Rodriguez, L. F., Torrelles, J. M., et al. 1989, ApJ, 341, 208

Boogert, A. C. A., Schutte, W. A., Tielens, A. G. G. M., et al. 1996, A\&A, 315, L377

Boogert, A. C. A., Helmich, F. P., van Dishoeck, E. F., et al. 1998, A\&A, 336, 352

Charnley, S. B., Whittet, D. C. B., \& Williams, D. A. 1990, MNRAS, 245,161

Chiar, J. E., Tielens, A. G. G. M., Whittet, D. C. B., et al. 2000, ApJ, 537,749

Churchwell, E., Walmsley, C. M., \& Cesaroni, R. 1990, A\&AS, 83, 119

Dartois, E., \& d'Hendecourt, L. 2001, A\&A, 365, 144

Dartois, E., Schutte W., Geballe, T., et al. 1999, A\&A, 342, L32

Demyk, K., Dartois, E., d'Hendecourt, L., et al. 1998, A\&A, 339, 553

d'Hendecourt, L., \& Allamandola, L. J. 1986, A\&AS, 64, 453 
d'Hendecourt, L., Joblin, C., \& Jones, A. (eds.) 1999, Solid Interstellar Matter: The ISO Revolution (Springer, Berlin; EDP Sciences, Les Ulis)

d'Hendecourt, L., Jourdain de Muizon, M., Dartois, E., et al. 1996, A\&A, 315, L365

Gerakines, P. A., Whittet, D. C. B., Ehrenfreund, P., et al. 1999, ApJ, 522,357

Gibb, E. L., Whittet, D. C. B., \& Chiar, J. E. 2001, ApJ, 558, 702

Gibb, E. L., Whittet, D. C. B., Schutte, W. A., et al. 2000, ApJ, 536, 347

Grim, R. J. A., \& Greenberg, J. M. 1987, ApJ, 321, L91

Graham, J. A., \& Chen, J W. P. 1991, AJ, 102, 1405

Grim, R. J. A., Baas, F., Greenberg, J. M., Geballe, T. R., \& Schutte, W. A. 1991, A\&A, 243, 473

Gürtler, J., Henning, Th., Kömpe, C., et al. 1996, A\&A, 315, L189

Hackwell, J. D., Gehrz, R. D., Smith, J. R., \& Briotta, D. A. 1978, ApJ, 221, 797

Hagen, W., Allamandola, L. J., \& Greenberg, J. M. 1980, A\&A, 86, L3

Hagen, W., Tielens, A. G. G. M., \& Greenberg, J. M. 1983, A\&AS, 51,389

Herbst, E. 1993, Gas-grain Chemistry in Quiescent Dense Interstellar Clouds, in Dust and Chemistry in Astronomy, ed. T. J. Millar, \& D. A. Williams (Institute of Physics Publ., Bristol), 9

Jijina, J., Myers, P. C., \& Adams, F. C. 1999, ApJS, 125, 161

Kerkhof, O., Schutte, W. A., \& Ehrenfreund, P. 1999, A\&A, 346, 990

Kessler, M. F., Müller, T. G., Arviset, C., García-Lario, P., \& Prusti, T. 2000, The ISO Handbook, Vol. I: ISO - Mission Overview, Version 1.0, November 24, 2001, SAI/2000-035/Dc (ESA publications), Villafranca

Kessler, M. F., Steinz, J. A., Anderegg, M. E., et al. 1996, A\&A, 315, L27

Klaas, U., Acosta-Pulido, J. A., Ábrahám, P., et al. 1997, ISOPHOT-S: Capabilities and Calibration, in Proc. First ISO Workshop on Analytical Spectroscopy, ed. A. M. Heras, K. Leech, N. R. Trams, \& M. Perry (ESA SP-419), 113

Klaas, U., Laureijs, R. J., Radovich, M., et al. 2000, ISOPHOT Calibration Accuracies, Vers. 4.0

Knacke, R. F., \& McCorkle, S. M. 1987, AJ, 94, 972
Knacke, R. F., McCorkle, S. M., Puetter, R. C., Erickson, E. F., \& Krätschmer, W. 1982, ApJ, 260, 141

Lacy, J. H., Baas, F., Allamandola, L. J., et al. 1984, ApJ, 276, 533

Lacy, J. H., Faraji, H., Sandford, S. A., \& Allamandola, L. J. 1998, ApJ, 501, L109

Lemke, D., Klaas, U., Abolins, J., et al. 1996, A\&A, 315, L64

Pendleton, Y. J., Tielens, A. G. G. M., Tokunaga, A. T., \& Bernstein, M. P. 1999, ApJ, 513, 294

Mauersberger, R., Wilson, T. L., Batrla, W., et al. 1985, A\&A, 146, 168

Salama, A., Kessler, M. F., Leech, K., \& Schulz, B. (eds.) 2000, ISO Beyond the Peaks: The 2nd ISO Workshop on Analytical Spectroscopy (ESA SP-456)

Sandford, S. A., Allamandola, L. J., \& Bernstein, M. P. 1998, Organic Chemistry: From the Interstellar Medium to the Solar System, in Origins, ed. C. E. Woodward, J. M. Shull, \& H. A. Thronson, Jr. (Astronomical Society of the Pacific, San Francisco), ASP Conf. Ser., 148, 392

Schutte, W. A., Tielens, A. G. G. M., Whittet, D. C. B., et al. 1996, A\&A, 315, L333

Skinner, C. J., Tielens, A. G. G. M., Barlow, M. J., \& Justtanont, K. 1992, ApJ, 399, L79

Smith, R. G., Sellgren, K., \& Tokunaga, A. T. 1989, ApJ, 344, 413

Sturm, E. 2000, How to reduce SWS AOT01 data in the ISAP GUI, ISO Explanatory Library Doc., http://www.ipac.caltech.edu/iso/sws/new_recipes/ sws01_recipe.ps

Sturm, E., Bauer, O. H., Brauer, J., et al. 1998, The ISO Spectral Analysis Package ISAP, Astronomical Data Analysis Software and Systems VII, ASP Conf. Ser., 145, ed. R. Albrecht, R. N. Hook, \& H. A. Bushouse, 161

Willner, S. P., Gillett, F. C., Herter, T. L., et al. 1982, ApJ, 253, 174

Whittet, D. C. B. 1993, Observations of Molecular Ices, in Dust and Chemistry in Astronomy, ed. T. J. Millar, \& D. A. Williams (Institute of Physics Publ., Bristol), 9

Whittet, D. C. B., Smith, R. G., Adamson, A. J., et al. 1996, ApJ, 458, 363 